\title{
Public Health Surveillance for the Great American Solar Eclipse in Oregon
}

\author{
Laurel Boyd ${ }^{\star 1}$, Meredith Jagger ${ }^{1}$, Kathryn Kuspis ${ }^{1}$, Melissa Powell ${ }^{1}$ and Sandy Giffin ${ }^{2}$ \\ 'Oregon Public Health Authority, Portland, OR, USA; ${ }^{2}$ Oregon Poison Center, Portland, OR, USA
}

\section{Objective}

Develop a public health surveillance plan for the Oregon Public Health Division (OPHD) in anticipation of the expected influx of visitors for the 2017 Great American Solar Eclipse.

\section{Introduction}

The Great American Solar Eclipse of 2017 provided a rare opportunity to view a complete solar eclipse on the American mainland. Much of Oregon was in the path of totality and forecasted to have clear skies. Ahead of the event, OPHD aggregated a list of 107 known gatherings in mostly rural areas across the state, some with estimated attendance of up to 30,000 attendees. Temporary food vendors and a range of sanitation solutions (including open latrines) were planned. International travelers were expected, along with large numbers of visitors traveling by car on the day of the eclipse.

The potential for multiple simultaneous mass gatherings across the state prompted OPHD to activate an incident management team (IMT) and to create a Health Intelligence Section to design a mass gathering surveillance strategy. Statewide syndromic surveillance (Oregon ESSENCE) has been used to monitor previous mass gatherings (1) and captures statewide emergency department (ED), urgent care, Oregon Poison Center, and reportable disease data.

\section{Methods}

The OPHD Health Intelligence Section identified five categories of public health effects associated with large outdoor gatherings based on literature review (2-5) and an internal risk assessment. These included health system status (total visit or call counts), communicable disease (fever, bloody diarrhea and reportable disease counts), injuries and substance abuse (including motor vehicle accidents), and outdoor exposure (heat-related illness, snake bites and asthma-related visits). An event-related category monitored trends in eclipse-related visits or eye-related injuries (looking directly at the sun). Where possible, syndromic trends were assessed in multiple data sources. These categories were used to create dashboards within Oregon ESSENCE and shared in a guidance document for local health departments and hospitals.

Health Intelligence monitored syndromes of interest during a period of enhanced surveillance $(9 / 18-9 / 22)$, and met daily with members of the OPHD IMT to share surveillance summaries, which were also sent to OPHD leadership and external partners.

\section{Results}

During the enhance surveillance period, the OPHD Health Intelligence Section did not identify statewide increases in healthcare utilization (total ED visits and calls to the Oregon Poison Center), but did observe increases in visits at select emergency departments in the state. Visits by out-of-state residents (as determined by patient zip code at time of registration) increased during the surveillance period. Fever-related visits increased as well but were not accompanied by reports of illnesses clusters.

Increases were noted for motor vehicle accidents, eye-related injuries, and "eclipse"-related visits. Increases in eye-related injuries appeared to be an annual seasonal trend and not related to the eclipse. There were no increases of note in the other queries monitored.
Development of new queries (West Nile Virus) was begun based upon mosquito pool surveillance findings. Surveillance highlights were posted publicly in a special edition of the biweekly Oregon ESSENCE Hazard Report (see Image 1).

\section{Conclusions}

Statewide public health surveillance during the 2017 Great American Solar Eclipse in Oregon did not identify clusters of infectious disease or other opportunities for real-time public health intervention. Nevertheless, surveillance identified increases in motor vehicle accidents, especially among out-of-state residents, due perhaps to increased road travel for the event. Preparations for this event increased capacity of state health department staff to conduct this type of surveillance in the future. Tools created for the eclipse have been used in several IMT activations since the eclipse.

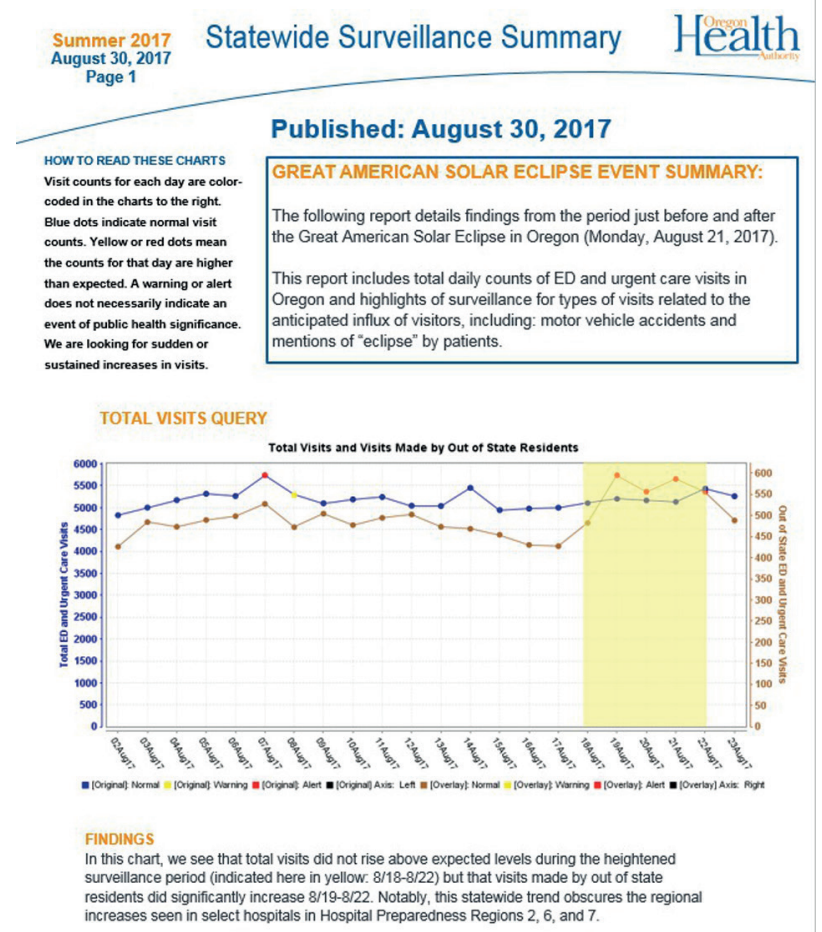

Portion of the 8/30/17 Oregon ESSENCE Hazard Report published online with surveillance findings from for the Great American Solar Eclipse

\section{Keywords}

mass gathering; syndromic surveillance; eclipse; incident command system

\section{Acknowledgments}

Tasha Poissant, Jamie Bash, Lisa Takeuchi, Alexia Zhang, Magdalena Scott, Roza Tammer, Emilio Debess, David Lehrfeld, Eric Gebbie, DeWayne Hatcher 


\section{ISDS 2018 Conference Abstracts}

References

1. Jagger MA, Jaramillo S, Boyd L, Johnson B, Reed KR, Powell M. Mass Gathering Surveillance : New ESSENCE Report and Collaboration Win Gold in OR. 2017;9(1):2579.

2. WHO. Public Health for Mass Gatherings: Key Considerations. World Health Organization. 2015.

3. Lombardo JS, Sniegoski CA, Loschen WA, Westercamp M, Wade M, Dearth S, et al. Public health surveillance for mass gatherings. Johns Hopkins APL Tech Dig (Applied Phys Lab. 2008;27(4):347-55.

4. Polkinghorne BG, Massey PD, Durrheim DN, Byrnes T, MacIntyre CR. Prevention and surveillance of public health risks during extended mass gatherings in rural areas: The experience of the Tamworth Country Music Festival, Australia. Public Health. 2013;127(1):32-8.

5. Burdick TE. Wilderness event medicine: Planning for mass gatherings in remote areas. Vol. 3, Travel Medicine and Infectious Disease. 2005. p. $249-58$

*Laurel Boyd

E-mail: laurelhifi@gmail.com 